\title{
Is behaving unethically for organizations a mixed blessing? A dual-pathway model for the work-to-family spillover effects of unethical pro-organizational behavior
}

\author{
Haixiao Chen ${ }^{1} \cdot$ Ho Kwong Kwan ${ }^{2}$ (D) Jie $\mathrm{Xin}^{3}$
}

Accepted: 21 May 2021 / Published online: 12 June 2021

(C) The Author(s) 2021

\begin{abstract}
This research examines the mixed work-to-family spillover effects of unethical proorganizational behavior. Drawing on conservation of resources theory and the workhome resources model, we develop a dual-pathway model to explain such effects. Based on a three-wave field study involving 214 respondents in China, we find engagement in unethical pro-organizational behavior to be positively associated with employees' organization-based self-esteem and stress at work, which in turn, leads to work-to-family positive spillover and work-to-family conflict, respectively. We also find that performing tensions moderate the mixed effects of unethical proorganizational behavior on organization-based self-esteem and work stress and the indirect effects of unethical pro-organizational behavior on work-to-family positive spillover and work-to-family conflict. Our findings have theoretical implications for business ethics scholars and practical implications for managers.
\end{abstract}

Keywords Unethical pro-organizational behavior · Performing tensions · Organizationbased self-esteem $\cdot$ Work stress $\cdot$ Work-to-family spillover

Jie Xin

cnxinjie@126.com

Haixiao Chen

chenhaixiao1218@foxmail.com

Ho Kwong Kwan

weicheong2317@hotmail.com

1 College of Business, Shanghai University of Finance and Economics, Shanghai 200433, People's Republic of China

2 Organizational Behavior and Human Resource Management Department, China Europe International Business School (CEIBS), Shanghai 201206, People's Republic of China

3 School of Management, Shandong University, Jinan, People's Republic of China 
Over the past decade, business ethics scholars have begun to systematically investigate and theorize about unethical pro-organizational behavior (UPB; Chen et al., 2016; Umphress et al., 2010). UPB involves "actions that are intended to promote the effective functioning of the organization or its members and violate core societal values, mores, laws, or standards of proper conduct" (Umphress \& Bingham, 2011: 622). Beyond assuming that unethical behavior is mainly driven by self-interest (e.g., Kish-Gephart et al., 2010; Mitchell et al., 2018), research on UPB has revealed that employees often engage in unethical behaviors to support the interests of their organization or its members (Umphress et al., 2010). Such behaviors may include intentionally covering-up product defects or misrepresenting the truth to embellish the organization's image. With increasingly in-depth studies on its antecedents, such as organizational identification (Chen et al., 2016), ethical leadership (Miao et al., 2013), and positive social exchange (Umphress \& Bingham, 2011), and its underlying mechanisms, such as moral disengagement (Chen et al., 2016) and neutralization (Umphress \& Bingham, 2011), knowledge about the factors leading to UPB has started to form.

Despite encouraging findings on the antecedents of UPB, little is known about its effects on the employees who engage in this type of unethical behavior. Given UPB's paradoxical nature being pro-social yet unethical (Tang et al., 2020; Umphress \& Bingham, 2011), a study on its effects is particularly intriguing and relevant. Research on ambivalence has revealed that "behaviors or events containing positive and negative elements" can elicit both positive and negative experiences (Rothman et al., 2017: 38). This suggests that the paradoxical nature of UPB may lead to mixed experiences (Tang et al., 2020). Theoretical work by UPB scholars, such as Umphress and Bingham (2011), has also suggested that the outcomes of UPB may be mixed. On the one hand, moral transgressions initiated to benefit others are expected to create positive meaning in the transgressor's perception, in contrast to self-interested unethical behavior (Levine \& Schweitzer, 2014; Wiltermuth, 2011). On the other hand, UPB is fundamentally unethical, and enactment of UPB may put employees at risk by exposing them to the possibility of future liability or other consequences (Moore \& Gino, 2015). Thus, UPB may act as a double-edged sword for employees who engage in it (Umphress \& Bingham, 2011).

Although sparse, recent studies have begun to explore the mixed effects of UPB. For example, Tang et al. (2020) found empirical evidence supporting the mixed effects of UPB in the work domain. Drawing on the appraisal theories of emotion, Tang et al. (2020) found that the paradoxical nature of UPB led to an experience of ambivalent emotion (i.e., both pride and guilt), resulting in increased citizenship behaviors towards the organization and customers. Yet, theoretical development and empirical evidence regarding the mixed effects of UPB remain scarce. The lack of research on the mixed effects of UPB prevents us from grasping the complexity of the phenomena of moral transgressions motivated by the desire to benefit an organization or its members, rather than the self (Umphress \& Bingham, 2011).

In this study, we attempt to extend the theoretical scope of the UPB literature beyond the work domain. Given that work and family roles are increasingly weighed up and interconnected in adult life (Kossek, 2006), the mixed effects of UPB may be farreaching. Current knowledge of behavioral ethics and prosocial behavior signifies the importance and legitimacy of understanding the link between UPB and work-family outcomes. In particular, the behavioral ethics literature has indicated that exposure to 
violations of moral principle affects ones' work-family experiences (e.g., Greenbaum et al., 2014; Liao et al., 2015). In addition, the prosocial behavior literature has suggested that going beyond the call of duty increases employees' feelings of job stress and subsequent work-family conflict (e.g., Bolino \& Grant, 2016; Bolino \& Turnley, 2005). The lack of investigation into the work-family outcomes of UPB may inhibit our understanding of its nature as both unethical and pro-social. By examining the work-to-family spillover effects of UPB, we not only explore its mixed effects, but also expand the nomological network concerning this type of moral transgression.

To do so, we build a dual-pathway model based on the work-home resources model (ten Brummelhuis \& Bakker, 2012) to connect UPB with work-to-family positive spillover (WFPS; Hanson et al., 2006) and work-to-family conflict (WFC; Greenhaus $\&$ Beutell, 1985). The work-home resources model integrates the core assumptions of conservation of resources (COR) theory (Hobfoll et al., 2018) and has been used to develop a theoretical dual-pathway explanation for resource spillover relationships between work and family (ten Brummelhuis \& Bakker, 2012). As indicated by COR theory, both gain and loss processes are related to resources conservation: in the former, resources are generated and accumulated, whereas in the latter, stress is developed and resources are depleted (Hobfoll et al., 2018). The work-home resources model provides a promising framework for exploring the mixed effects of UPB and allows us to combine its resource-generating and resource-depleting processes. Specifically, we propose that engagement in UPB indirectly promotes WFPS by enhancing organization-based self-esteem (OBSE), an important personal resource in the workplace (Kwan et al., 2020; ten Brummelhuis \& Bakker, 2012). Additionally, UPB intensifies the inter-role conflict between the work and family domains (Greenhaus \& Beutell, 1985) through the experience of work stress, which captures the resources loss at work (Hobfoll et al., 2018).

To further understand the boundary condition of the mixed effects of UPB, we tested the potential moderating role of performing tensions. Under the rubric of organizational tensions, performing tensions denote the paradoxes faced by employees when fostering their multiple and competing performance goals in an organization, such as being flexible while complying with the organization's rules (Miron-Spektor et al., 2018). The concept of performing tensions is highly associated with but fundamentally different from both role conflict (i.e., the certain sense of incompatibility between role requirements) and role ambiguity (i.e., the lack of sufficiently clear information to accomplish a role; Rizzo et al., 1970). Theoretically, performing tensions have been widely examined in the paradox literature (Smith \& Lewis, 2011), whereas role conflict and ambiguity were developed by role theory (Rizzo et al., 1970). People's conceptualization of tensions affects the impacts of those tensions. Perception of tensions indicates that employees can describe the competing demands placed on them without necessarily experiencing conflict or ambiguity. In other words, an employee who does not embrace the paradoxes or tensions is likely to experience conflict or ambiguity. These mixed effects of UPB reflect the complex or even paradoxical reality of UPB performance. This contextual factor may clarify why in some circumstances, UPB is more likely to be interpreted as acceptable conduct and thus is more beneficial and less detrimental.

This study makes three main contributions to the business ethics literature. First, we present our dual-pathway model, established to examine the work-to-family effects of 
UPB. We contribute to the UPB literature by examining the possibility that partaking in UPB may both benefit and harm the actor and by extending the research scope from the work domain to the family domain. Second, we enrich the understanding of the paradoxical nature of UPB by exploring the mechanisms linking UPB and family outcomes based on the work-home resources model. By integrating both its resourcegenerating and resource-depleting processes, we can examine the mixed effects of UPB and explain why UPB leads to increased OBSE and work stress. Finally, we contribute to both UPB and work-family research by identifying performing tensions as a boundary condition in relation to our dual-pathway model. The moderating role of performing tensions also has potential implications in terms of an organization's ability to regulate the effects of UPB. To test our proposed dual-pathway model, we conduct a three-wave field survey in China involving a sample of 214 part-time Master of Business Administration (MBA) students.

\section{Theory and hypothesis development}

\section{Indirect effect of UPB on WFPS through OBSE}

This study drew mainly on the work-home resources model to account for the effect of UPB on the work-family interface. The work-home resources model suggests that through interactions at work, people acquire certain personal resources that enrich their family lives and suppress conflict between work and family (ten Brummelhuis \& Bakker, 2012). In other words, personal resources comprise an important mediating mechanism that explains the relationship between work behaviors and the work-family interface.

UPB might help employees to accumulate personal resources. In the work domain, one important personal resource is OBSE (Greenhaus \& Powell, 2006; ten Brummelhuis \& Bakker, 2012), which refers to "the self-perceived value that individuals have of themselves as organization members acting within an organizational context" (Pierce et al., 1989: 625). OBSE represents a positive view of self that can be activated by successful experiences within the organization or by contributions made within a domain in which a person has invested a sense of self (Pfeffer \& Fong, 2005; Taylor \& David, 1996). Fostering OBSE makes an employee feel "capable, significant, and worthy as an organization member” (Pierce \& Gardner, 2004: 593).

We argue that enactment of UPB is positively associated with an employee's OBSE. Research has indicated that UPB can elicit the experience of positive emotions, such as pride (Tang et al., 2020). Pride is considered a primary self-conscious emotion that is "linked to self-esteem regulation and maintenance" and "gives self-esteem its affective kick" (Tracy \& Robins, 2007: 506). When individuals have a sense of "specific accomplishments", their pride can be elicited and an increase in self-worth is accompanied (Tracy \& Robins, 2007: 507). Employees initiate UPB to improve the functioning of their organization, even though their actions may violate moral norms or sacrifice their social interests (Umphress \& Bingham, 2011; Warren, 2003). Such actions of in-group favoritism help employees to attain a sense of accomplishment (Lewis, 1993), which entails a perception of self-worth in the organization (Martiny \& Rubin, 2016). In addition, as UPB represents "heroic sacrifices" to perform unethical 
acts that benefit the organization (Chen et al., 2013; Thau et al., 2015: 107), employees are likely to take credit for engaging in UPB and to consider themselves as valuable and worthy members in the organization. In other words, the enactment of UPB allows an employee to maintain a self-representation such as "I am a good and contributive employee for the organization" (Tang et al., 2020). This is consistent with one of the core principles of COR theory, which proposes that the investment of resources facilitates the process of resources gaining (Hobfoll et al., 2018). Thus, we propose:

Hypothesis 1 Engaging in UPB is positively associated with OBSE.

We further propose that OBSE should positively affect the WFPS. WFPS reflects the extent of spillover, in which one's resources gains at work are transferred into the family domain (Edwards \& Rothbard, 2000; Hanson et al., 2006). The results of previous studies suggested that self-esteem nurtured in a work role can increase performance in the family role (for a review, see Greenhaus \& Powell, 2006). There are two main explanations of this relationship between OBSE and WFPS (e.g., Edwards \& Rothbard, 2000; Greenhaus \& Powell, 2006). First, the positive feelings associated with OBSE, such as a sense of accomplishment, can be transferred to the home environment, which leads to positive affects in the family domain (Edwards \& Rothbard, 2000). Second, spillover occurs when an employee with enhanced OBSE considers themself to be valuable and capable (Bowling et al., 2010). This selfperception helps to expand their thought-action repertoires and outward focus of attention (Fredrickson \& Branigan, 2005; Wang et al., 2021; Yang et al., 2018). These expansions then stimulate active interactions with family members and confident engagement with family tasks, thus enabling a positive spillover from work to family (Carlson et al., 2014).

According to the work-home resources model (ten Brummelhuis \& Bakker, 2012), partaking in UPB could increase an employee's WFPS through OBSE via two mechanisms. On the one hand, UPB elevates OBSE and the transfer of positive affects from work to home (Isen \& Baron, 1991). Employees can directly share these positive affects with family members in their home environments, thereby increasing their WFPS (Greenhaus \& Powell, 2006). On the other hand, UPB makes a contribution to the organization or its members beyond the limits of moral norms. This elevates the actor's OBSE and thus improves their thought-action repertoires and outward focus of attention (Fredrickson \& Branigan, 2005; Yang et al., 2018). At home, an employee who experiences self-enhancement after practicing UPB is likely to pay close attention to and care for their family members. Taken together, we propose the following:

Hypothesis 2 UPB and WFPS are positively and indirectly related via OBSE, such that UPB is positively associated with OBSE and OBSE is positively associated with WFPS.

\section{Indirect effect of UPB on WFC through work stress}

However, engagement in UPB might also deplete personal resources in the work domain. This resources loss at work can be captured by the construct of work stress, 
which represents the conscious or subconscious psychological responses of an individual to resource-depleting situations (Hobfoll, 1989, 2001; LePine et al., 2004). The conservation of resources theory indicates that work stress can fall on employees who are losing (or at risk of losing) resources (e.g., time and energy; Hobfoll, 1989, 2001).

We presume that engaging in UPB is positively linked to an employee's perception of work stress. Recent empirical evidence has shown that behaviors enacted for others can deplete the actor's resources (e.g., Koopman et al., 2016; Lanaj et al., 2016). As UPB is initiated by the employee in favor of the organization or its members (Umphress \& Bingham, 2011), its regulation may require a heavy investment of personal resources, which would place the actor in a stressful situation (Hobfoll et al., 2018). In addition, research on emotion has indicated that employees who engage in UPB experience ambivalent emotions at work (Tang et al., 2020), namely the "simultaneous existence of strong, polar opposite feelings" (Rothman et al., 2017: 35 ), such as a bittersweet feeling. The very experience of emotional ambivalence can make an employee "feel torn and conflicted" (Rothman et al., 2017: 35), leading to the depletion of resources. Thus, although engaging in UPB might enhance an employee's experience of an accomplishment and their self-value within the organization, it is also likely to increase stress in their work domain.

Furthermore, people are socialized to experience discomfort after violating the norms held by their larger community (Lagattuta \& Thompson, 2007). The expectations and perceptions of what constitutes ethically appropriate behaviors in the workplace are crucial for whether stress is experienced (Edwards, 1992; Zhong et al., 2010). As UPB improves the performance of the overall organization or other members by violating societal moral norms and inflicting potential or actual public costs (Thau et al., 2015; Umphress \& Bingham, 2011), those who engage in such behavior face the risk of exposure and future liability. Considering that UPB involves activities that are initiated by employees themselves and "neither ordered by a supervisor nor part of a formal job description" (Lee et al., 2019: 109), the employees are more likely to hold themselves responsible for their conduct and potential negative consequences (Tang et al., 2020; Tangney et al., 2007). The anxiety of being responsible for the potential consequences, such as reputation damage or after-sale complaints from customers (Bonner et al., 2017; Cialdini et al., 2004), threatens the employee's well-being and requires the use of resources for coping (Cooper et al., 2019), which leads to work stress. Accordingly, we propose the following hypothesis:

Hypothesis 3 Engaging in UPB is positively associated with work stress.

Employees often face challenges in balancing their multiple roles (Kossek, 2006). WFC refers to "a form of inter-role conflict" in which role pressures from the work domain affect the employee's performance in the family domain (Greenhaus \& Beutell, 1985: 77). This is commonly viewed as a work-to-family negative spillover (Carlson et al., 2000; Frone et al., 1992). The resources perspective has been used widely to explain WFC (e.g., Edwards \& Rothbard, 2000; Greenhaus \& Beutell, 1985). As individuals have finite resources that can be expended immediately to handle multiple roles, it is difficult for them to freely satisfy all of the demands and expectations of each role (Frone et al., 1992; Michel et al., 2011). The devotion of resources to the work 
domain will leave the individual less able to meet the requirements and expectations of the family domain, thereby leading to WFC (Greenhaus \& Beutell, 1985).

Along these lines, this study applies the resources perspective to the relation between work stress and WFC (Hobfoll, 1989, 2001; ten Brummelhuis \& Bakker, 2012). Often, work stress plays a resource-depleting role in the work-family relationship and limits an employee's ability to fulfil their family role. For example, work stress could undermine an employee's capacity to work effectively (Xin et al., 2018). Consequently, more time may be needed for work tasks, leaving less time to fulfil family responsibilities. Work stress also drain's the employee's energy, which is a critical personal resource in relation to their capacity to fulfil their family responsibilities. WFC is more likely to occur when such resources are lacking. This positive relationship between work stress and WFC has been corroborated by several meta-analysis studies (e.g., Byron, 2005; Michel et al., 2011).

According to the work-home resources model (ten Brummelhuis \& Bakker, 2012), we propose that UPB increases the conflict between work and family via work stress. Specifically, work stress links UPB and WFC by interfering with the finite resources needed to fulfil both work and family responsibilities. Such resource depletion makes it more difficult for employees to fulfil their family role responsibilities, resulting in WFC (ten Brummelhuis \& Bakker, 2012). For instance, an employee who lies to customers by exaggerating or misrepresenting the product quality is placed under a high level of stress. At home, these employees are likely to be fatigued and to avoid family activities (Greenhaus \& Beutell, 1985). Therefore, we propose the following:

Hypothesis 4 UPB and WFC are positively and indirectly related via work stress, such that UPB is positively associated with work stress, and work stress is positively associated with WFC.

\section{Moderating role of performing tensions}

Organizations are inundated with pervasive tensions and competing demands, including performing, learning, belonging, and organizing tensions (Smith \& Lewis, 2011). An examination of the type of tensions depends on the context of study (Miron-Spektor et al., 2018). As this study was not conducted during a dynamic system change (when learning tensions surface), did not involve a dyadic or group-level analysis (when belonging tensions surface), and was not related to creating competing designs and processes at work (when organizing tensions surface), we focused only on performing tensions, which arise from the differing and often competing demands at work (Smith \& Lewis, 2011). Performing tensions reflect the paradoxical demands at work for fostering organizational success (Miron-Spektor et al., 2018; Smith \& Lewis, 2011), which stem from paradoxical performance goals and irreconcilable expectations. For instance, in an organization with high performing tensions, employees may be required to be flexible when responding to an encountered problem while complying with the organization's rules (Miron-Spektor et al., 2018).

Organizational tensions represent ambiguous and complex configurations of reality pervading a person's organizational life (Miron-Spektor et al., 2018). Given their paradoxical nature, these tensions not only increase employees' sense of uncertainty 
and threaten their sense of order (e.g., Lewis, 2000; Vince \& Broussine, 1996), but also equip them with the awareness to cope with paradoxes and look for approaches to achieve competing performance goals (Miron-Spektor et al., 2018; Schneider, 1999). Consequently, individuals who experience tensions devote efforts to interpreting and making sense of the situation (Weick, 1995), which implies that organizational tensions also affect how individuals look upon and evaluate organizational behaviors. In comparison to the resistance or avoidance of tensions, the performance of flexible actions or behaviors in a tense environment is more likely to be recognized or appreciated by the organization and its members (Lüscher \& Lewis, 2008; Schneider, 1999). We argue that these performing tensions can help to justify UPB and thus facilitate the beneficial effects of UPB while buffering against its destructive effects.

In particular, performing tensions strengthen the positive link from UPB to OBSE. According to self-enhancement theory, an individual can selectively perceive or construe ambiguous information to make themself more accomplished and capable (Kunda \& Rasyid, 1989), while ignoring or avoiding information that threatens their positive self-conception (Sedikides \& Green, 2000). As performing tensions signal ambiguity, employees who engage in UPB are prone to highlight their efforts in terms of facilitating the organization's functions and to neglect the immoral aspect of these behaviors (Thau et al., 2015; Umphress \& Bingham, 2011). To wit, in an organization with performing tensions, employees are more likely to perceive and construe their UPB in a positive light and thus regard themselves as valuable and meaningful organizational members.

Hypothesis 5 Performing tensions moderate the positive relationship between UPB and OBSE, such that this relationship is stronger when the level of performing tensions is high, rather than low.

In addition, employees who perform UPB also suffer less from work stress when the level of performing tensions is high. Therefore, we argue that employees in such situations consider UPB to be justifiable. Performing tensions require paradoxical performance achievements, which threaten the employee's sense of order (MironSpektor et al., 2018; Vince \& Broussine, 1996) and increase their uncertainty regarding what the organization needs them to achieve. In these situations, employees tend to engage in work activities based on their own interpretations about how to reach their performance goals and contribute to the organization. As UPB is composed of selfinitiated actions intended to contribute to the organization beyond a supervisor's orders or a formal job description (Lee et al., 2019), unethical behaviors can be considered a "flexible" way to improve the organization's performance, or even a sign of a willingness to risk the self for the sake of the organization (Thau et al., 2015). Therefore, performing UPB in an organization with high levels of performing tensions is less likely to be experienced as a threat and to cause work stress (Karasek, 1979; Lazarus \& Folkman, 1984). On this basis, we propose the following hypothesis:

Hypothesis 6 Performing tensions moderate the positive relationship between UPB and work stress, such that this relationship is weaker when the level of performing tensions is high than when it is low. 
Above, we have hypothesized a dual-pathway model from UPB to the work-family interface and explained how performing tensions moderate the effects of UPB on OBSE and work stress. Given that OBSE and work stress mediate the work-family effects of UPB, we argue that both effects are moderated by performing tensions. That is, with higher OBSE and lower work stress experienced under high performing tensions, the positive spillover effect of UPB on WFPS via OBSE would be strengthened and the negative spillover effect of UPB on WFC via work stress would be alleviated. Therefore, we propose a moderated mediation model (Edwards \& Lambert, 2007) in which performing tensions moderate the mixed work-family spillover effects of UPB.

Hypothesis 7 Performing tensions moderate the indirect effect of UPB on WFPS through OBSE, such that the indirect effect is stronger when performing tensions are high than when they are low.

Hypothesis 8 Performing tensions moderate the indirect effect of UPB on WFC through work stress, such that the indirect effect is weaker when performing tensions are high than when they are low.

\section{Method}

\section{Sample and procedures}

The study participants were part-time Master of Business Administration (MBA) students recruited from a university in Shandong province, China. They were all working full time in a range of companies in both the public and private sectors. Questionnaires were coded in such a way as to guarantee anonymity, and we distributed the questionnaires with a covering letter assuring confidentiality and that data would be used solely for academic research purposes. To ensure that the participants paid attention to all of the items in the questionnaires, attention checks were used in all three waves.

To prevent potential problems associated with common method bias (Podsakoff et al., 2003), we collected the survey data in three waves at one-month intervals. This temporal separation approach was recommended by Podsakoff et al. (2003) as a way to alleviate common method bias by helping to "reduce the respondent's ability to infer missing details by allowing previously recalled information to leave short-term memory" (Podsakoff et al., 2003: 888). To select the appropriate time interval lengths, we reviewed the UPB and work-family interface literature based on time-lagged research designs and observed a wide variation in the selected time intervals for data collection, ranging from two weeks (e.g., Chen et al., 2016) to three months (e.g., Loi et al., 2018). As we proposed and tested a moderated mediation model to reveal the process and contingent effects of UPB, we adopted a relatively medium-length time interval (one month), which allowed the participants to perceive and evaluate their statuses in relation to resources gain and depletion.

In our first-wave survey (T1), the participants were asked to assess their UPB and levels of performing tensions at work and to answer nine demographic questions on their gender, age, education, tenure, position, marital status, number of children, and 
whether they lived with a spouse, parents, and children. We sent 354 questionnaires to potential participants; of these, 297 were returned, for a response rate of $83.90 \%$. For the second-wave survey (T2), the participants who had returned the first-wave questionnaire were administered questionnaires asking them to assess their OBSE and work stress during the previous month. Of the 297 surveys sent, 245 were retrieved, for a response rate of $82.49 \%$. One month after the second survey, participants who had returned the second-wave questionnaire were sent third-wave questionnaires (T3) in which they were asked to assess their WFPS and WFC during the previous month. Of the 245 surveys sent, 240 useful responses were received, giving a response rate of $97.95 \%$. After checking and matching the questionnaires, our final sample consisted of 214 respondents, with an overall response rate of $60.45 \%$.

Overall, $43.50 \%$ of the respondents were female, and they had a mean of 12.30 years of work experience $(S D=9.97)$. Moreover, $62.10 \%$ were frontline employees or managers and $37.90 \%$ were mid-level or higher managers. The mean age was 34.61 years $(S D=7.67)$, and $72.40 \%$ of the participants were married. Almost three quarters $(73.80 \%)$ of the respondents had a bachelor's degree, and $19.20 \%$ had a master's or higher degree. In addition, $143(66.80 \%)$ of the participants lived with their spouse, $57(26.60 \%)$ lived with their parents, $123(57.50 \%)$ had more than one child, and $101(47.20 \%)$ lived with their children.

\section{Measures}

Scales Unless otherwise stated, we measured each item on a 5-point Likert scale $(1=$ strongly disagree to $5=$ strongly agree). UPB was measured using a 6 -item scale that was developed by Umphress et al. (2010) and applied to the Chinese context by Chen et al. (2016). We asked each respondent to indicate the degree to which they agreed with each statement associated with the current situation of their organization. An example item in this scale is, "If it would help my organization, I would misrepresent the truth to make my organization look good." The reliability of this scale was .71 . Performing tensions was measured using a 3-item scale originally developed by MironSpektor et al. (2018). Because the measurement of performing tensions had not been tested in the Chinese context, we translated the scale according to the translation/ backtranslation procedure (Brislin, 1986) to ensure equivalency. An example scale item is, "In my work, I need to be flexible while also complying with the company's tight rules." The reliability of this scale was .67. We measured OBSE using a 10-item scale that was developed by Pierce et al. (1989) and used by Hui and Lee (2000) in China. An example item on this scale is, "I am an important part of this place." The reliability of this scale was .90. Work stress was measured using the anxiety-stress scale developed by House and Rizzo (1972), which contains 26 items. For this study, we adopted the simplified 4-item scale used by Netemeyer et al. (2005). An example item is, "At the end of the day, my work leaves me stressed out." The reliability of this scale was .78. WFPS was measured using an 11-item scale that was developed by Hanson et al. (2006) and used in a Chinese setting by Liu et al. (2012). An example item is, "Having a good day at work allows me to be optimistic with my family." The reliability of this scale was .91 . WFC was measured using a 9-item scale that was developed by Carlson et al. (2000) and adopted by Tang et al. (2016) in a Chinese setting. An 
example item is, "I am often so emotionally drained when I get home from work that it prevents me from contributing to my family." The reliability of this scale was .93.

Control variables Based on previous work-family literature (e.g., Barnett \& Hyde, 2001; Friedman \& Greenhaus, 2000; Greenhaus \& Powell, 2006), we controlled for 10 demographic variables: gender $(1=$ female and $2=$ male), age (in years), education ( $1=$ middle school or below to $5=$ Master's degree or above), job tenure (in years), position $(1=$ frontline employee to $5=$ director $)$, marital status $(1=$ married and $2=$ single), number of children, living with spouse, living with parents, and living with children (each coded as $1=$ yes, $2=$ no, and $3=$ not applicable). We created two dummy variables for each of these last three control variables - six dummy variables altogether - in line with the structural equation modeling process. We also controlled the mediating effects of organizational identification, which has strongly captured the attention of UPB scholars (e.g., Chen et al., 2016; Umphress et al., 2010). In particular, research has indicated that engagement in UPB may improve an actor's organizational identification (e.g., Umphress \& Bingham, 2011). In this study, we applied the 6-item organizational identification scale that was originally developed by Mael and Ashforth (1992) and later adopted by Zhang et al. (2012) in a Chinese setting. An example item is, "When someone criticizes my organization, it feels like a personal insult." The reliability of the scale was .88 .

\section{Results}

\section{Descriptive statistics}

The descriptive statistics and correlations of all of the variables are presented in Table 1. The results of the correlation analyses provide a preliminary overview of the relationships between the variables.

\section{Testing the measurement model}

To estimate the construct validity of the measures and the distinctiveness of the six key variables (UPB, performing tensions, OBSE, work stress, WFPS and WFC) and one control variable (organizational identification), we conducted a series of confirmatory factor analyses using Mplus 8.0 (Muthén \& Muthén, 2017). Given the small ratio of the sample size to the parameters for estimation in our model (Bentler \& Chou, 1987), we applied item-parceling procedures to reduce the number of estimated parameters (Landis et al., 2000). Specifically, the number of indicators on each unidimensional scale (i.e., UPB, performing tensions, organizational identification, OBSE, and work stress) was parceled to a more parsimonious three per factor according to the singlefactor method suggested by Landis et al. (2000). The multi-dimensional scales (i.e., WFPS and WFC) were treated as second-order factors in the model. Table 2 presents the results of the model fit estimation. The proposed 7-factor model (M1) yielded a good measurement fit $\left(\chi^{2}[d f=533]=924.77, p<.01, \mathrm{RMSEA}=.06, \mathrm{CFI}=.93\right.$, and 


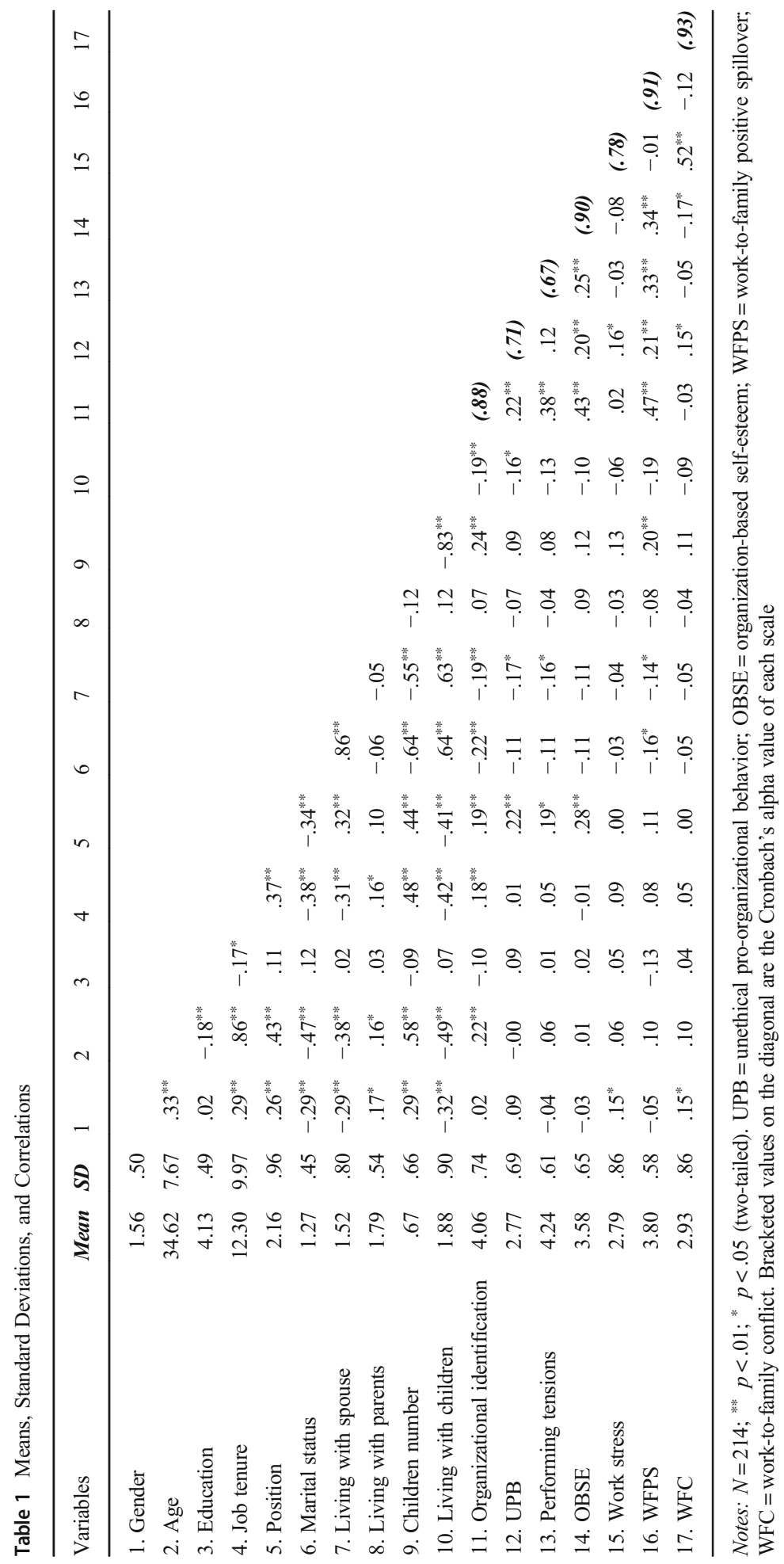


Table 2 Results of Confirmatory Factor Analyses

\begin{tabular}{|c|c|c|c|c|c|c|}
\hline Model & $x^{2}$ & $d f$ & RMSEA & CFI & TLI & $\Delta \chi^{2}(d f)$ \\
\hline M1: The measurement model: Seven factors & 924.77 & 533 & .06 & .93 & .92 & \\
\hline $\begin{array}{l}\text { M2: UPB and performing tensions were combined as } \\
\text { one factor }\end{array}$ & 1063.47 & 539 & .07 & .90 & .89 & $138.70^{* *}(6)^{\mathrm{a}}$ \\
\hline $\begin{array}{l}\text { M3: OBSE and work stress were combined as one } \\
\text { factor }\end{array}$ & 1061.12 & 539 & .07 & .88 & .87 & $136.35^{* *}(6)^{\mathrm{a}}$ \\
\hline M4: WFPS and WFC were combined as one factor & 3187.54 & 545 & .15 & .50 & .45 & $2262.77^{* *}(12)^{\mathrm{a}}$ \\
\hline $\begin{array}{l}\text { M5: All constructs loaded on a higher-order factor: } \\
\text { CMV }\end{array}$ & 1021.32 & 547 & .07 & .91 & .90 & $96.55^{* *}(14)^{\mathrm{a}}$ \\
\hline $\begin{array}{l}\text { M6: Full mediation model: Work stress was allowed to } \\
\text { be correlated with OBSE }\end{array}$ & 1441.86 & 944 & .05 & .91 & .90 & \\
\hline $\begin{array}{l}\text { M7: Nested partial mediation model: Adding paths } \\
\text { from UPB to WFPS and UPB to WFC }\end{array}$ & 1439.14 & 942 & .05 & .91 & .90 & $2.72(2)^{\mathrm{b}}$ \\
\hline
\end{tabular}

Notes: $N=214 ;{ }^{* *} p<.01 ;{ }^{*} p<.05$. RMSEA: root mean square error of approximation, CFI: comparative fit index, TLI: Tucker-Lewis index

${ }^{a}$ Model was compared with the measurement model

${ }^{\mathrm{b}}$ Model was compared with M6

TLI =.92). All of the factor loadings were significant, supporting the convergent validity.

To estimate the distinctiveness of the constructs, we applied model comparison procedures in which the hypothesized measurement model (M1) was compared with several alternative models. Specifically, we contrasted M1 against a six-factor model (M2) that combined the key constructs of T1 (i.e., UPB and performing tensions), a sixfactor model (M3) that combined the key constructs of T2 (i.e., OBSE and work stress), and a six-factor model (M4) that combined the key constructs of T3 (i.e., WFPS and WFC). The results of the chi-square tests shown in Table 2 show that the hypothesized measurement model (M1) yielded a significantly better fit than any of the alternatives. The discriminant validity was thus confirmed. In addition, to test the potential for common method bias, we loaded all of the key constructs on a higher-order latent factor for treatment as a common method variance (CMV) factor. The model fit of this common method model (M5: $\chi^{2}[d f=547]=1021.32, p<.01$, RMSEA $=.07$, $\mathrm{CFI}=.91$, and $\mathrm{TLI}=.90)$ was significantly inferior to that of the measurement model (M1; $\left.\Delta \chi^{2}[\Delta d f=14]=96.55, p<.01\right)$. Thus, our results were not likely to be significantly influenced by common method bias.

\section{Testing of structural models}

We then tested our hypotheses by applying latent structural equation modeling (LSEM) via Mplus 8.0 (Muthén \& Muthén, 2017). To test Hypotheses 1 and 3, we regressed OBSE and work stress, respectively, on UPB and the 10 demographic variables. The observed significant positive relationships of UPB with OBSE $(\beta=.19, S E=.08$, $p<.05)$ and work stress $(\beta=.24, S E=.10, p<.05)$ supported Hypotheses 1 and 3. 
To test the mediating effects of OBSE and work stress as proposed in Hypotheses 2 and 4, we first estimated a full mediation model (M6), in which WFPS and WFC were simultaneously regressed on OBSE, work stress, organizational identification, and the 10 demographic variables, while organizational identification, OBSE and work stress were simultaneously regressed on UPB and the 10 demographic variables. This model (M6) yielded a good model fit $\left(\chi^{2}[d f=663]=1056.42, p<.01\right.$, RMSEA $=.06$, $\mathrm{CFI}=.91$, and TLI $=.90)$. We then compared the full mediation model (M6) with our measurement model (M1) and a theoretically nested partial mediation model (M7), which was adapted from M6 by adding direct paths from UPB to WFPS and from UPB to WFC. According to the results of a chi-square test (shown in Table 2), the full mediation model (M6) fitted the data better than M7 $\left(\Delta \chi^{2}[\Delta d f=2]=2.72\right.$, n.s. $)$. Moreover, the results obtained from M7 indicated that the direct paths from UPB to WFPS $(\beta=.07, S E=.04, p=.11)$ and WFC $(\beta=.12, S E=.09, p=.15)$ were not significant. Thus, we selected M6 as the optimal model for testing the proposed mediating effects in this study (see Table 2).

In M6, we tested Hypotheses 2 and 4 using both a $t$-test and bootstrapping approach. The $95 \%$ bootstrapping confidence intervals (CIs) of the latent indirect effects of OBSE and work stress were estimated using the Monte Carlo method with 20,000 replications (Preacher et al., 2010). The results of the $t$-test and bootstrapping test were highly consistent. Specifically, after controlling the mediating effects of organizational identification, the mediating effect of OBSE in the relationship between UPB and WFPS was significant (indirect effect $=.03, S E=.01,95 \%$ CI $[.01, .08]$, excluding zero), consistent with Hypothesis 2. The mediating effect of work stress on the relationship between UPB and WFC was also significant (indirect effect $=.15, S E=.07,95 \% \mathrm{CI}$ $[.02, .33]$, excluding zero), supporting Hypothesis 4 . Table 3 presents the results of the latent indirect effects.

Table 3 Result of latent conditional indirect effects of performing tensions

\begin{tabular}{lccc}
\hline Relationships & $\beta$ & $S E$ & $95 \%$ CIs \\
\hline UPB $\rightarrow$ OBSE $\rightarrow$ WFPS & .03 & .01 & {$[.01, .08]$} \\
Mean level & & & \\
Conditional indirect effect: & -.01 & .01 & {$[-.04, .03]$} \\
Low performing tensions (-1SD) & .04 & .02 & {$[.01, .09]$} \\
High performing tensions (+1 SD) & .05 & .02 & {$[.01, .10]$} \\
Difference & & & .07 \\
UPB $\rightarrow$ Work Stress $\rightarrow$ WFC & .15 & & {$[.02, .33]$} \\
Mean level & & .09 & {$[.10, .46]$} \\
Conditional indirect effect: & .26 & .09 & {$[-.09, .27]$} \\
Low performing tensions $(-1 S D)$ & .09 & .08 & {$[-.36,-.02]$} \\
High performing tensions $(+1 S D)$ & -.17 & & \\
Difference & & & \\
\hline
\end{tabular}

Notes: Bootstrapping $=20,000$. UPB = unethical pro-organizational behavior; OBSE = organization-based self-esteem; WFPS = work-to-family positive spillover; $\mathrm{WFC}=$ work-to-family conflict; $\mathrm{CI}=$ confidence interval 


\section{Testing moderation and the moderated mediation model}

Hypotheses 5 and 6 propose the moderating role of performing tensions in the relationships of UPB $\rightarrow$ OBSE and UPB $\rightarrow$ Work Stress. To test these hypotheses, we created a moderated mediation model based on the full mediation model (M6), which included the latent interaction between UPB and performing tensions. After applying the numerical integration technique (Klein \& Moosbrugger, 2000), the results (shown in Fig. 1) indicated that the latent interaction had a significant effect on OBSE $(\beta=.25, S E=.11, p<.05)$ and work stress $(\beta=-.14, S E=.06, p<.05)$. Figures 2 and 3 present the plots of simple slope tests based on performing tensions with values that were 1 standard deviation above and below the mean, respectively. The graphical results are consistent with our hypotheses. Thus, Hypotheses 5 and 6 were supported by our empirical data.

Hypotheses 7 and 8 propose first-stage moderating effects of performing tensions on the relationships of $\mathrm{UPB} \rightarrow \mathrm{OBSE} \rightarrow$ WFPS and UPB $\rightarrow$ Work Stress $\rightarrow$ WFC. Table 3 presents the results of the latent conditional indirect effects by applying the Monte Carlo bootstrapping approach with 20,000 replications (Preacher et al., 2010). Specifically, UPB had a significant indirect effect on WFPS via OBSE when performing tensions were at a high level $(+1 S D$; conditional indirect effect $=.04, S E=.02,95 \% \mathrm{CI}$ $[.01, .09]$, excluding zero). In contrast, this indirect relationship was non-significant when performing tensions were at a low level $(-1 S D$; conditional indirect effect= $-.01, S E=.01,95 \%$ CI $[-.04, .03]$, including zero). Furthermore, the difference between the two groups was significant (between-group differences $=.05, S E=.02$, 95\% CI [.01, .10], excluding zero), which supported Hypothesis 7. Moreover, the indirect effect of UPB on WFC via work stress was significant when performing tensions were at a low level $(-1 S D$; conditional indirect effect $=.26, S E=.09,95 \%$ CI $[.10, .46]$, excluding zero) but non-significant when performing tensions were at a high level (+1 SD; conditional indirect effect $=.09, S E=.09,95 \%$ CI $[-.09, .27]$, including zero). This difference was also significant (between-group differences = $-.17, S E=.08,95 \%$ CI $[-.36,-.02]$, excluding zero), supporting Hypothesis 8 . Therefore, our proposed moderated mediation model is supported by the data.

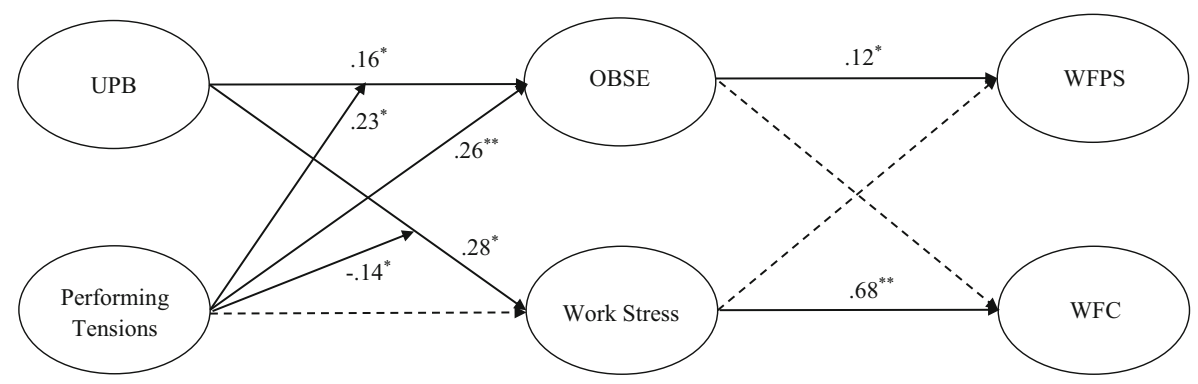

Fig. 1 Structural equation modeling results for moderated mediation model. Notes: $N=214$; ${ }^{* *} p<.01$, * $p<.05$ (two-tailed). UPB = unethical pro-organizational behavior; OBSE = organization-based self-esteem; WFPS = work-to-family positive spillover; WFC = work-to-family conflict. The numbers are coefficients of each path. Solid line indicates significant path, and dashed line indicates nonsignificant path 


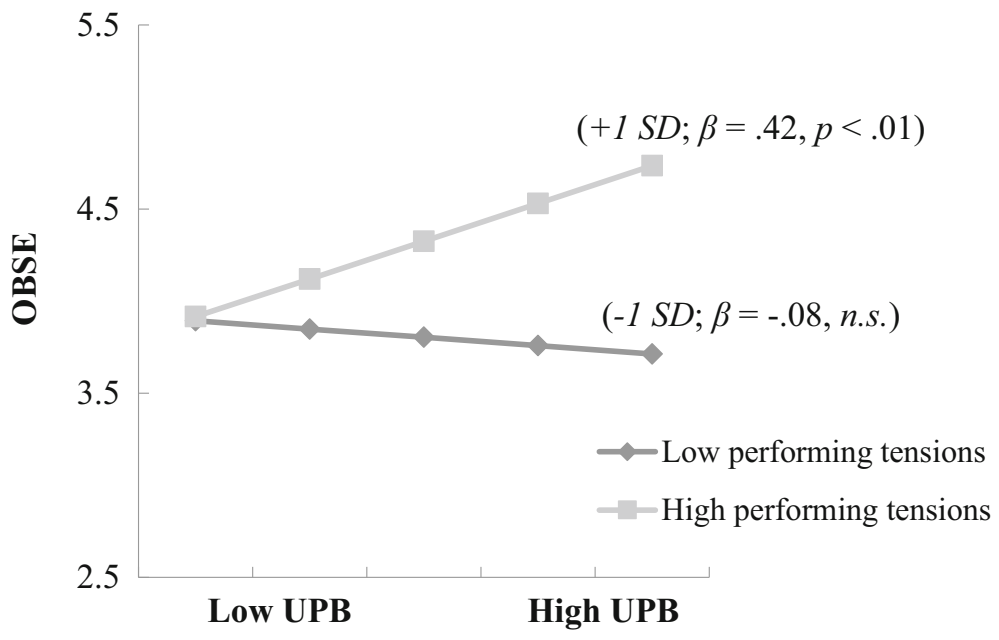

Fig. 2 The moderating role of performing tensions in the relationship between UPB and OBSE

\section{Discussion}

To further a comprehensive understanding of the reflexive effects of unethical behavior initiated to benefit an organization (UPB), we built a dual-pathway model and tested it via a three-wave field survey. Our results showed that UPB exerts mixed effects on the work-family interface, leading to WFPS and WFC. OBSE and work stress are mechanisms underlying the UPB-WFPS and UPB-WFC relations, respectively. Furthermore, our results support the hypothesis that performing tensions within an organization are a critical moderator of these mixed effects of UPB.

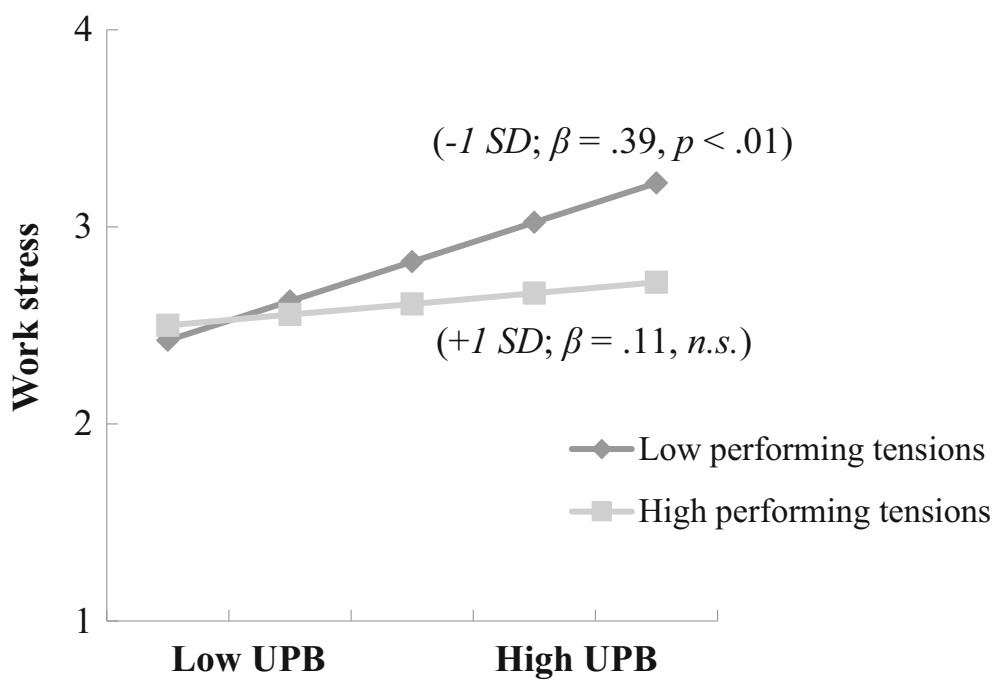

Fig. 3 The moderating role of performing tensions in the relationship between UPB and work stress 


\section{Theoretical implications}

Our research makes three noteworthy contributions. First, our findings contribute to the business ethics literature by advancing knowledge about the mixed effects of UPB across the work and family domains and showing that the mixed effects of UPB can be far-reaching. Behavioral ethics scholars have overwhelmingly focused on either the outcome variables of self-serving unethical behavior (e.g., Kish-Gephart et al., 2010; Mitchell et al., 2018; Ruedy et al., 2013) or the antecedents of UPB (e.g., Chen et al., 2016; Lee et al., 2019; Miao et al., 2013; Umphress \& Bingham, 2011). In contrast, research into the outcomes of UPB is scant. In their theoretical model, Umphress and Bingham (2011) suggested that engaging in UPB may be a mixed blessing. The results of more recent empirical research by Tang et al. (2020) primarily supported this suggestion and showed that UPB elicits ambivalent emotions in actors, leading to mixed outcomes at work. In this study, we developed a dual-pathway model and determined that the mixed effects of UPB are transferrable across the work and family domains. Although a large body of research has examined the work outcomes of unethical behavior at work (e.g., Bonner et al., 2017; Shalvi et al., 2015), little attention has been paid to the potential effects of these unethical acts on employees in their nonwork domains. As work and family roles are highly weighed and interconnected in adult life (Kossek, 2006), our findings enrich the body of knowledge about the effects of UPB (e.g., Umphress \& Bingham, 2011) by showing that such behavior can increase WFPS and WFC.

Second, our research uses the resources perspective to enrich our understanding of the mechanisms underlying the mixed work-family effects of UPB. Previously, our understanding of UPB was mainly fostered and largely expanded by social cognitive theory (e.g., moral disengagement; Chen et al., 2016; Umphress \& Bingham, 2011), social exchange theory (e.g., positive reciprocity beliefs; Umphress et al., 2010), and emotional perspective (e.g., pride and guilt; Tang et al., 2020). However, few studies explored UPB from the resource perspective, especially in relation to resources spillover across domains. In this study, we developed a dual-pathway model by drawing on the work-home resources model (ten Brummelhuis \& Bakker, 2012), which allowed us to combine the resource-generating and resource-depleting processes of UPB and explain the mixed effects of UPB from the work domain to the family domain. By utilizing the work-home resources model (ten Brummelhuis \& Bakker, 2012), we accounted for the influences of UPB on employees' family lives via OBSE and work stress. This is an intriguing finding because it implies that employees who partake in UPB may not only perceive themselves as important members of the organization but may also experience high levels of stress at work, which would have mixed effects on their work-family interfaces. Thus, our study extends the UPB literature by exploring the mixed nature of UPB from a resources perspective, providing an avenue for future research on the mixed effects of UPB.

Third, our investigation on the moderating role of organizational tensions extends the UPB research and provides a new angle on the organizational management of the mixed effects of UPB. The existing UPB literature provides evidence for moderators on the effects of predictors on performing UPB (e.g., Chen et al., 2016; Umphress et al., 2010). This study, however, revealed performing tensions as a possible boundary condition that could influence the mixed effects of UPB. Specifically, a high level of 
performing tensions in an organization can benefit employees who engage in UPB by rendering their behavior more acceptable and justifiable. UPB scholars have called for more research in the organizational context to provide a foundation for employees' perceptions about "what is and what is not the appropriate behavior within the organization" (Umphress \& Bingham, 2011: 629). As the mixed effects of UPB reflect the complex, or even paradoxical, reality of practicing UPB (Tang et al., 2020), our work specifically enriches the body of UPB research by incorporating performing tensions into our dual-pathway model. This inclusion helps us to understand the complexity of the mixed effects of UPB (Miron-Spektor et al., 2018).

Further, our investigation of the moderating effects of performing tensions provides some interesting insights with respect to the work-family literature. Specifically, positive work-to-family spillover caused by UPB might be facilitated, and negative spillover inhibited in an organization with a high level of paradox. This indicates that to some extent, tensions affect an employee's approach to handling their work-family relationship. Employees often face challenges in balancing the requirements of multiple roles (Kossek, 2006), and many of which are paradoxical. Our findings suggest that tensions in an organization may benefit the work-family relationship by facilitating the work-to-family spillover processes of some organizational behaviors.

\section{Managerial implications}

Our findings also have implications for organizations and managers. Although the direct effects of UPB might be beneficial to the organization because of its proorganizational aspect, we suggest that organizations should be vigilant against the unethical aspect of such moral transgressions (Cialdini et al., 2004). Even though an organization may benefit from its employees' UPB in the short term, it may ultimately experience undesirable consequences in the form of societal sanctions or a long-term decline in financial performance (Weaver et al., 1999). Our findings showed that engaging in UPB is a double-edged sword at the individual level, indicating that enactment of UPB is not performed without personal costs. Employees who engage in UPB not only perceive enhanced self-worth within the organization, but also regard their work stressful. Thus, managers should pay attention to the extent to which employees engage in unethical conducts in the name of the organization.

Despite the efforts made by organizations to manage unethical phenomena (Treviño et al., 2014), unethical behavior is becoming increasingly prevalent (Bazerman \& Tenbrunsel, 2011; Bonner et al., 2017). When UPB occurs, managers should act to reduce the associated damage and prevent undesirable consequences. Our empirical findings on the moderating effects of performing tensions suggest that an organization can manipulate and manage the effects of engaging in UPB on its employees. Particularly, our findings indicate that employees who engage in UPB can benefit from tensions within the organization, as this helps them to develop OBSE and avoid taking on additional work stress. To meet the goal of inhibiting employees from engaging in UPB, it is important to avoid magnifying the benefits of such behavior. Therefore, we suggest that organizations take action to adjust their employees' perceptions of UPB by managing organizational tensions.

Recent findings from paradox research suggest that organizations can manage tensions through several approaches. First, tensions should be apprehended correctly 
in the workplace. For example, Miron-Spektor et al. (2018) argued that "the problem is how we think about the problem" and suggested that particular cognitive frames (i.e., paradox mindset) can facilitate paradox identification and management. Second, tensions may be solvable by reducing the asymmetry between capacity and expectations. For example, Li (2021a, 2021b) suggested that paradoxes can be alleviated by increasing one's capacities, decreasing one's expectations, or doing both simultaneously. Nevertheless, Berti and Simpson (2021) warned that organizations should pay attention to power asymmetry, which may twist their efforts to manage tensions. Third, the paradox literature has underlined the important role of leaders in tension management. For example, Zhang et al. (2015) indicated that organizations should focus on paradoxical leader behaviors that help to balance the tensions between organizations' structural needs and employees' individual needs.

\section{Limitations and future directions}

This study has several limitations. First, we believe that self-report measures capture a broader range of unethical behaviors than other-report measures, as some unethical behaviors are not conducted in front of others (Berry et al., 2012; Umphress et al., 2010). However, the self-reporting of behavior may increase the potential for common method variance (Podsakoff et al., 2003). To alleviate these concerns, we used a timelagged research design (Doty \& Glick, 1998) in which the collection of variablesindependent, mediating, and dependent — was separated into three waves. The results of a statistic remedy analysis (M5; Liu et al., 2015; Williams \& McGonagle, 2016) showed that our findings had a low susceptibility to common method bias. Nevertheless, in the future, researchers should aim to collect data associated with supervisor- or peer-derived ratings of UPB.

Second, we acknowledge that we could not confirm causal relationships in this study because of our time-lagged research design (Law et al., 2016). For example, employees with a high OBSE might plausibly violate moral norms for the benefit of the organization or its members because they may feel more responsible for the development and welfare of the organization. Although our theoretical developments support a general spillover process from UPB to WFPS, our time-lagged research design did not allow us to confirm whether UPB increases OBSE or vice versa. Therefore, an experimental or longitudinal research design should be implemented in the future to provide more robust evidence for the causal relations in our model.

Third, differences in the cultural valuation of virtues (e.g., Miller \& Bersoff, 1992; Waytz et al., 2013) and the sociocognitive systems (e.g., Hofstede, 2001; Nisbett et al., 2001) may have potential influences on our findings for the following reasons. First, given that East Asian societies place a higher value on loyalty than Western societies (Hofstede, 2001; Waytz et al., 2013) behaviors that violate moral norms but contribute to the collective are more likely to be prevalent and acceptable, and to be positively associated with the perceived importance of group membership in the Chinese context. Second, according to the literature on sociocognitive systems (e.g., Fang, 2012; Nisbett et al., 2001), a traditional Western approach derived from the laws of Aristotle's formal logic and of Hegelian logic emphasizes the separation and distinction of competing elements when dealing with tensions (Leung et al., 2018). In contrast, the traditional Eastern approach is derived from Yin-Yang theory and East Asian ancient dialectical 
thoughts and encourages the acceptance and integration of competing elements (Fang, 2012). People from an Eastern culture may be more adaptive to contradiction and tolerant of ambiguity (Schad \& Bansal, 2018). Thus, the positive aspect of performing tensions might be amplified in a Chinese sample. In this context, our participants may have perceived performing tensions as an opportunity rather than a neutral situation. We suggest that future research should pay more attention to the cultural implications of UPB.

Fourth, our findings indicate that contextual factors, specifically, paradoxical performance goals made by the organization, could moderate the mixed effects of UPB. However, we did not examine other potential moderators, such as the employees' personalities (e.g., dark triads; Castille et al., 2018), ethical leadership (Miao et al., 2013), and ethical norms at work (e.g., egoistic norms, Graham et al., 2020). For instance, narcissism, one of the dark triad personalities that reflects the extent of the desire to maintain an exceedingly positive sense of self (Morf \& Rhodewalt, 1993), may moderate the positive relationship between UPB and OBSE. Miao et al. (2013) proposed the important role of ethical leadership in triggering UPB. Plausibly, ethical leadership could make employees less vulnerable to the benefits of UPB. In addition, the opportunity to engage in UPB and the consequent reaction may be different for employees working in the private and public sectors. For example, employees in the public sector (e.g., government institutions) are responsible for public affairs and act in the public's interest, rather than the organization's interest (Lawton et al., 2013), suggesting that UPB is less likely to be appraised in the public sector. Therefore, we recommend that UPB scholars examine additional potential moderators of the effects of UPB.

Finally, the low reliability of the performing tensions measure is noteworthy. The scale used to measure this variable had a relatively low level of reliability in both our study and the literature in which it was originally developed (Miron-Spektor et al., 2018). Although the performing tensions scale has a strong theoretical basis (i.e., paradox theory; Smith \& Lewis, 2011) and it was the first relatively reliable scale to capture the concept of performing tensions in the organization, further endeavors are needed to improve the measurement of this variable. Paradox scholars have argued that it is difficult to capture all types of organizational tensions because each can exhibit various specific forms (e.g., Smith \& Lewis, 2011). For example, employees' interpretations of performing tensions may vary according to the requirements of different competing goals. Therefore, we suggest that future research should pay attention to the specific context in which specific forms of tensions emerge.

\section{Conclusion}

The present study investigated the outcomes of employees who engage in unethical conduct initiated in favor of an organization or its members (i.e., UPB). By drawing upon the COR theory and work-home resources model, we built a dual-pathway theoretical model to examine the mixed work-to-family effects of UPB. Our hypothesized model was supported by a data analysis of a three-wave field survey study. Our findings indicated that in the positive spillover path, UPB promotes WFPS via enhanced OBSE, whereas in the negative spillover path, UPB intensifies WFC via 
increased work stress. In addition, the indirect path from UPB to WFPS is enhanced by performing tensions, whereas the indirect path from UPB to WFC is mitigated by performing tensions. Our work thus contributes to the literature on behavioral ethics by exploring employees' responses to the practice of UPB and the mixed effects of this type of behavior on the work and family domains.

Acknowledgements The authors would like to thank Norihiko Takeuchi and the two anonymous reviewers for their very helpful comments and suggestions.

Funding This study was funded by the National Natural Science Foundation of China (grant numbers 71672108, 71972118).

Open Access This article is licensed under a Creative Commons Attribution 4.0 International License, which permits use, sharing, adaptation, distribution and reproduction in any medium or format, as long as you give appropriate credit to the original author(s) and the source, provide a link to the Creative Commons licence, and indicate if changes were made. The images or other third party material in this article are included in the article's Creative Commons licence, unless indicated otherwise in a credit line to the material. If material is not included in the article's Creative Commons licence and your intended use is not permitted by statutory regulation or exceeds the permitted use, you will need to obtain permission directly from the copyright holder. To view a copy of this licence, visit http://creativecommons.org/licenses/by/4.0/.

\section{References}

Barnett, R. C., \& Hyde, J. S. 2001. Women, men, work, and family: An expansionist theory. American Psychologist, 56: 781-796.

Bazerman, M. H., \& Tenbrunsel, A. E. 2011. Blind spots: Why we fail to do what's right and what to do about it. Princeton University Press.

Bentler, P. M., \& Chou, C. P. 1987. Practical issues in structural modeling. Sociological Methods and Research, 16: 78-117.

Berry, C. M., Carpenter, N. C., \& Barratt, C. L. 2012. Do other-reports of counterproductive work behavior provide an incremental contribution over self-reports? A meta-analytic comparison. Journal of Applied Psychology, 97: 613-636.

Berti, M., \& Simpson, A. V. 2021. The dark side of organizational paradoxes: The dynamics of disempowerment. Academy of Management Review, 16: 252-274.

Bolino, M. C., \& Grant, A. M. 2016. The bright side of being prosocial at work, and the dark side, too: A review and agenda for research on other-oriented motives, behavior, and impact in organizations. The Academy of Management Annals, 10: 599-670.

Bolino, M. C., \& Turnley, W. H. 2005. The personal costs of citizenship behavior: The relationship between individual initiative and role overload, job stress, and work-family conflict. Journal of Applied Psychology, 90: 740-748.

Bonner, J. M., Greenbaum, R. L., \& Quade, M. J. 2017. Employee unethical behavior to shame as an indicator of self-image threat and exemplification as a form of self-image protection: The exacerbating role of supervisor bottom-line mentality. Journal of Applied Psychology, 102: 1203-1221.

Bowling, N. A., Eschleman, K. J., Wang, Q., Kirkendall, C., \& Alarcon, G. 2010. A meta-analysis of the predictors and consequences of organization-based self-esteem. Journal of Occupational and Organizational Psychology, 83: 601-626.

Brislin, R. W. 1986. The wording and translation of research instrument. In W. Lonner \& J. Berry (Eds.), Field methods in cross-cultural research: 137-164. Sage.

Byron, K. 2005. A meta-analytic review of work-family conflict and its antecedents. Journal of Vocational Behavior, 67: 169-198. 
Carlson, D. S., Hunter, E. M., Ferguson, M., \& Whitten, D. 2014. Work-family enrichment and satisfaction mediating processes and relative impact of originating and receiving domains. Journal of Management, 40: 845-865.

Carlson, D. S., Kacmar, K. M., \& Williams, L. J. 2000. Construction and initial validation of a multidimensional measure of work-family conflict. Journal of Vocational Behavior, 56: 249-276.

Castille, C. M., Buckner, J. E., \& Thoroughgood, C. N. 2018. Prosocial citizens without a moral compass? Examining the relationship between machiavellianism and unethical pro-organizational behavior. Journal of Business Ethics, 149: 919-930.

Chen, M., Chen, C. C., \& Sheldon, O. J. 2016. Relaxing moral reasoning to win: How organizational identification relates to unethical pro-organizational behavior. Journal of Applied Psychology, 101: 1082-1096.

Chen, Y., Ferris, D. L., Kwan, H. K., Yan, M., Zhou, M., \& Hong, Y. 2013. Self-love's lost labor: A selfenhancement model of workplace incivility. Academy of Management Journal, 56: 1199-1219.

Cialdini, R. B., Petrova, P. K., \& Goldstein, N. J. 2004. The hidden costs of organizational dishonesty. MIT Sloan Management Review, 45: 67-74.

Cooper, B., Wang, J., Bartram, T., \& Cooke, F. L. 2019. Well-being-oriented human resource management practices and employee performance in the Chinese banking sector: The role of social climate and resilience. Human Resource Management, 58: 85-97.

Doty, D. H., \& Glick, W. H. 1998. Common methods bias: Does common methods variance really bias results? Organizational Research Methods, 1: $374-406$.

Edwards, J. R. 1992. A cybernetic theory of stress, coping, and well-being in organizations. Academy of Management Review, 17: 238-274.

Edwards, J. R., \& Lambert, L. S. 2007. Methods for integrating moderation and mediation: A general analytical framework using moderated path analysis. Psychological Methods, 12: 1-22.

Edwards, J. R., \& Rothbard, N. P. 2000. Mechanisms linking work and family: Clarifying the relationship between work and family constructs. Academy of Management Review, 25: 178-199.

Fang, T. 2012. Yin Yang: A new perspective on culture. Management and Organization Review, 8: 25-50.

Fredrickson, B. L., \& Branigan, C. A. 2005. Positive emotions broaden the scope of attention and thoughtaction repertoires. Cognition \& Emotion, 19: 313-332.

Friedman, S., \& Greenhaus, J. 2000. Work and family - allies or enemies? Oxford University Press.

Frone, M. R., Russell, M., \& Cooper, M. L. 1992. Antecedents and outcomes of work-family conflict: Testing a model of the work-family interface. Journal of Applied Psychology, 77: 65-78.

Graham, K. A., Resick, C. J., Margolis, J. A., Shao, P., Hargis, M. B., \& Kiker, J. D. 2020. Egoistic norms, organizational identification, and the perceived ethicality of unethical pro-organizational behavior: A moral maturation perspective. Human Relations, 73: 1249-1277.

Greenbaum, R. L., Quade, M. J., Mawritz, M. B., Kim, J., \& Crosby, D. 2014. When the customer is unethical: The explanatory role of employee emotional exhaustion onto work-family conflict, relationship conflict with coworkers, and job neglect. Journal of Applied Psychology, 99: 1188-1203.

Greenhaus, J. H., \& Beutell, N. J. 1985. Sources of conflict between work and family roles. Academy of Management Review, 10: 76-88.

Greenhaus, J. H., \& Powell, G. N. 2006. When work and family are allies: A theory of work-family enrichment. Academy of Management Review, 31: 72-92.

Hanson, G. C., Hammer, L. B., \& Colton, C. L. 2006. Development and validation of a multidimensional scale of perceived work-family positive spillover. Journal of Occupational Health Psychology, 11: 249265.

Hobfoll, S. E. 1989. Conservation of resources: A new attempt at conceptualizing stress. American Psychologist, 44: 513-524.

Hobfoll, S. E. 2001. The influence of culture, community, and the nested-self in the stress process: Advancing conservation of resources theory. Applied Psychology, 5: 337-421.

Hobfoll, S. E., Halbesleben, J., Neveu, J.-P., \& Westman, M. 2018. Conservation of resources in the organizational context: The reality of resources and their consequences. Annual Review of Organizational Psychology and Organizational Behavior, 5: 103-128.

Hofstede, G. H. 2001. Culture's consequences: Comparing values, behaviors, institutions and organizations across nations. Beverly Hills, CA: Sage.

House, R. J., \& Rizzo, J. R. 1972. Role conflict and ambiguity as critical variables in a model of organizational behavior. Organizational Behavior and Human Performance, 7: 467-505.

Hui, C., \& Lee, C. 2000. Moderating effects of organization-based self-esteem on organizational uncertainty: Employee response relationships. Journal of Management, 26: 215-232. 
Isen, A. M., \& Baron, R. A. 1991. Positive affect as a factor in organizational behavior. In B. M. Staw \& L. L. Cummings (Eds.), Research in Organizational Behavior: 1-53. Greenwich, CT: JAI Press.

Karasek, R. A. 1979. Job demands, job decision latitude, and mental strain: Implications for job redesign. Administrative Science Quarterly, 24: 285-308.

Kish-Gephart, J. J., Harrison, D. A., \& Treviño, L. K. 2010. Bad apples, bad cases, and bad barrels: Metaanalytic evidence about sources of unethical decisions at work. Journal of Applied Psychology, 95: 1-31.

Klein, A., \& Moosbrugger, H. 2000. Maximum likelihood estimation of latent interaction effects with the LMS method. Psychometrika, 65: 457-474.

Koopman, J., Lanaj, K., \& Scott, B. A. 2016. Integrating the bright and dark sides of OCB: A daily investigation of the benefits and costs of helping others. Academy of Management Journal, 59: 414-435.

Kossek, E. E. 2006. Work and family in America: Growing tension between employment policy and a transformed workforce. In E. Lawler \& J. O'Toole (Eds.), America at work: Choices and challenges: 5372. NY: Palgrave MacMillan.

Kunda, Z., \& Rasyid, S. 1989. Motivated changes in the self concept. Journal of Experimental Social Psychology, 25: 272-285.

Kwan, H. K., Chen, H., \& Chiu, R. K. 2020. Effects of empowering leadership on followers' work-family interface. The International Journal of Human Resource Management, 1-34.

Lagattuta, K. H., \& Thompson, R. A. 2007. The development of self-conscious emotions: Cognitive processes and social influences. In J. L. Tracy, R. W. Robins \& J. P. Tangney (Eds.), The self-conscious emotions: Theory and research: 91-113. New York, NY: Guilford Press.

Lanaj, K., Johnson, R. E., \& Wang, M. 2016. When lending a hand depletes the will: The daily costs and benefits of helping. Journal of Applied Psychology, 101: 1097-1110.

Landis, R. S., Beal, D. J., \& Tesluk, P. E. 2000. A comparison of approaches to forming composite measures in structural equation models. Organizational Research Methods, 3: 186-207.

Law, K. S., Wong, C.-S., Yan, M., \& Huang, G. 2016. Asian researchers should be more critical: The example of testing mediators using time-lagged data. Asia Pacific Journal of Management, 33: 319-342.

Lawton, A., Rayner, J., \& Lasthuizen, K. 2013. Ethics and management in the public sector. Routledge.

Lazarus, R. S., \& Folkman, S. 1984. Stress, appraisal, and coping. New York: Springer.

Lee, A., Schwarz, G., Newman, A., \& Legood, A. 2019. Investigating when and why psychological entitlement predicts unethical pro-organizational behavior. Journal of Business Ethics, 154: 109-126.

LePine, J. A., LePine, M. A., \& Jackson, C. L. 2004. Challenge and hindrance stress: Relationships with exhaustion, motivation to learn, and learning performance. Journal of Applied Psychology, 89: 883-891.

Leung, A. K., Liou, S., Miron-Spektor, E., Koh, B., Chan, D., Eisenberg, R., \& Schneider, I. 2018. Middle ground approach to paradox: Within- and between-culture examination of the creative benefits of paradoxical frames. Journal of Personality and Social Psychology, 114: 443-464.

Levine, E. E., \& Schweitzer, M. E. 2014. Are liars ethical? On the tension between benevolence and honesty. Journal of Experimental Social Psychology, 53: 107-117.

Lewis, M. W. 1993. Self-conscious emotions: Embarrassment, pride, shame, and guilt. In M. Lewis \& J. M. Haviland (Eds.), Handbook of emotions: 563-573. New York, NY, US: Guilford Press.

Lewis, M. W. 2000. Exploring paradox: Toward a more comprehensive guide. Academy of Management Review, 25: 760-776.

Li, X. 2021a. Quantum approach to organizational paradox: A copenhagen perspective. Academy of Management Review, 46: 412-415.

Li, X. 2021b. Solving paradox by reducing expectation. Academy of Management Review, 46: 409-412.

Liao, Y., Liu, X.-Y., Kwan, H. K., \& Li, J. 2015. Work-family effects of ethical leadership. Journal of Business Ethics, 128: 535-545.

Liu, J., Kwan, H. K., \& Mao, Y. 2012. Mentorship quality and protégés' work-to-family positive spillover, career satisfaction and voice behavior in China. International Journal of Human Resource Management, 23: 4110-4128.

Liu, S., Luksyte, A., Zhou, L., Shi, J., \& Wang, M. 2015. Overqualification and counterproductive work behaviors: Examining a moderated mediation model. Journal of Organizational Behavior, 36: 250-271.

Loi, R., Xu, A. J., Chow, C. W. C., \& Kwok, J. M. L. 2018. Customer misbehavior and store managers' workto-family enrichment: The moderated mediation effect of work meaningfulness and organizational affective commitment. Human Resource Management, 57: 1039-1048.

Lüscher, L. S., \& Lewis, M. W. 2008. Organizational change and managerial sensemaking: Working through paradox. Academy of Management Journal, 51: 221-240.

Mael, F., \& Ashforth, B. E. 1992. Alumni and their alma mater: A partial test of the reformulated model of organizational identification. Journal of Organizational Behavior, 13: 103-123. 
Martiny, S. E., \& Rubin, M. 2016. Towards a clearer understanding of social identity theory's self-esteem hypothesis. In S. McKeown, R. Haji \& N. Ferguson (Eds.), Understanding Peace and Conflict Through Social Identity Theory: 19-32. Springer International Publishing.

Miao, Q., Newman, A., Yu, J., \& Xu, L. 2013. The relationship between ethical leadership and unethical proorganizational behavior: Linear or curvilinear effects? Journal of Business Ethics, 116: 641-653.

Michel, J. S., Kotrba, L. M., Mitchelson, J. K., Clark, M. A., \& Baltes, B. B. 2011. Antecedents of workfamily conflict: A meta-analytic review. Journal of Organizational Behavior, 32: 689-725.

Miller, J. G., \& Bersoff, D. M. 1992. Culture and moral judgment: How are conflicts between justice and interpersonal responsibilities resolved? Journal of Personality and Social Psychology, 62: 541-554.

Miron-Spektor, E., Ingram, A., Keller, J., Smith, W. K., \& Lewis, M. W. 2018. Microfoundations of organizational paradox: The problem is how we think about the problem. Academy of Management Journal, 61: 26-45.

Mitchell, M. S., Baer, M. D., Ambrose, M. L., Folger, R., \& Palmer, N. F. 2018. Cheating under pressure: A self-protection model of workplace cheating behavior. Journal of Applied Psychology, 103: 54-73.

Moore, C., \& Gino, F. 2015. Approach, ability, aftermath: A psychological process framework of unethical behavior at work. The Academy of Management Annals, 9: 235-289.

Morf, C. C., \& Rhodewalt, F. 1993. Narcissism and self-evaluation maintenance: Explorations in object relations. Personality and Social Psychology Bulletin, 19: 668-676.

Muthén, L. K., \& Muthén, B. O. 2017. Mplus user's guide (8th ed.). Los Angeles, CA: Author.

Netemeyer, R. G., Iii, J. G. M., \& Pullig, C. 2005. Conflicts in the work-family interface: Links to job stress, customer service employee performance, and customer purchase intent. Journal of Marketing, 69: 130143.

Nisbett, R. E., Peng, K., Choi, I., \& Norenzayan, A. 2001. Culture and systems of thought: Holistic versus analytic cognition. Psychological Review, 108: 291-310.

Pfeffer, J., \& Fong, C. T. 2005. Building organization theory from first principles: The self-enhancement motive and understanding power and influence. Organization Science, 16: 372-388.

Pierce, J. L., \& Gardner, D. G. 2004. Self-esteem within the work and organizational context: A review of the organization-based self-esteem literature. Journal of Management, 30: 591-622.

Pierce, J. L., Gardner, D. G., Cummings, L. L., \& Dunham, R. B. 1989. Organization-based self-esteem: Construct definition, measurement, and validation. Academy of Management Journal, 32: 622-645.

Podsakoff, P. M., MacKenzie, S. B., Lee, J.-Y., \& Podsakoff, N. P. 2003. Common method biases in behavioral research: A critical review of the literature and recommended remedies. Journal of Applied Psychology, 88: 879-903.

Preacher, K. J., Zyphur, M. J., \& Zhang, Z. 2010. A general multilevel SEM framework for assessing multilevel mediation. Psychological Methods, 15: 209-233.

Rizzo, J. R., House, R. J., \& Lirtzman, S. I. 1970. Role conflict and ambiguity in complex organizations. Administrative Science Quarterly, 15: 150-163.

Rothman, N. B., Pratt, M. G., Rees, L., \& Vogus, T. J. 2017. Understanding the dual nature of ambivalence: Why and when ambivalence leads to good and bad outcomes. Academy of Management Annals, 11: 3372.

Ruedy, N. E., Moore, C., Gino, F., \& Schweitzer, M. E. 2013. The cheater's high: The unexpected affective benefits of unethical behavior. Journal of Personality and Social Psychology, 105: 531-548.

Schad, J., \& Bansal, P. 2018. Seeing the forest and the trees: How a systems perspective informs paradox research. Journal of Management Studies, 55: 1490-1506.

Schneider, K. J. 1999. The paradoxical self: Toward an understanding of our contradictory nature. New York: Insight Books.

Sedikides, C., \& Jeffrey, D. G. 2000. On the self-protective nature of inconsistency-negativity management: Using the person memory paradigm to examine self-referent memory. Journal of Personality and Social Psychology, 79: 906-922.

Shalvi, S., Gino, F., Barkan, R., \& Ayal, S. 2015. Self-serving justifications: Doing wrong and feeling moral. Current Directions in Psychological Science, 24: 125-130.

Smith, W. K., \& Lewis, M. W. 2011. Toward a theory of paradox: A dynamic equilibrium model of organizing. Academy of Management Review, 36: 301-403.

Tang, G., Kwan, H. K., Zhang, D., \& Zhu, Z. 2016. Work-family effects of servant leadership: The roles of emotional exhaustion and personal learning. Journal of Business Ethics, 137: 285-297.

Tang, P. M., Kai, C. Y., \& Joel, K. 2020. Feeling proud but guilty? Unpacking the paradoxical nature of unethical pro-organizational behavior. Organizational Behavior and Human Decision Processes, 160: 68-86. 
Tangney, J. P., Stuewig, J., \& Mashek, D. J. 2007. Moral emotions and moral behavior. Annual Review of Psychology, 58: 345-372.

Taylor, S. E., \& David, A. A. 1996. Positive illusions and coping with adversity. Journal of Personality, 64: 873-989.

ten Brummelhuis, L. L., \& Bakker, A. B. 2012. A resource perspective on the work-home interface: The work-home resources model. American Psychologist, 67: 545-556.

Thau, S., Derfler-Rozin, R., Pitesa, M., Mitchell, M. S., \& Pillutla, M. M. 2015. Unethical for the sake of the group: Risk of social exclusion and pro-group unethical behavior. Journal of Applied Psychology, 100: 98-113.

Tracy, J. L., \& Robins, R. W. 2007. The psychological structure of pride: A tale of two facets. Journal of Personality and Social Psychology, 92: 506-525.

Treviño, L. K., den Nieuwenboer, N. A., \& Kish-Gephart, J. J. 2014. (Un)ethical behavior in organizations. Annual Review of Psychology, 65: 635-660.

Umphress, E. E., \& Bingham, J. B. 2011. When employees do bad things for good reasons: Examining unethical pro-organizational behaviors. Organization Science, 22: 621-640.

Umphress, E. E., Bingham, J. B., \& Mitchell, M. S. 2010. Unethical behavior in the name of the company: The moderating effect of organizational identification and positive reciprocity beliefs on unethical proorganizational behavior. Journal of Applied Psychology, 95: 769-780.

Vince, R., \& Broussine, M. 1996. Paradox, defense and attachment: Accessing the working with emotions and relations underlying organizational change. Organizational Studies, 17: 1-21.

Wang, Q., Liao, Y., \& Burns, G. N. 2021. General, work-specific, and work-role conscientiousness measures in predicting work criteria: A comparative perspective. Applied Psychology: An International Review, 70: 358-383.

Warren, D. E. 2003. Constructive and destructive deviance in organizations. Academy of Management Review, 28: 622-632.

Waytz, A., Dungan, J., \& Young, L. 2013. The whistleblower's dilemma and the fairness-loyalty tradeoff. Journal of Experimental Social Psychology, 49: 1027-1033.

Weaver, G. R., Treviño, L. K., \& Cochran, P. L. 1999. Corporate ethics programs as control systems: Influences of executive commitment and environmental factors. Academy of Management Journal, 42: 41-57.

Weick, K. E. 1995. Sensemaking in organizations. Thousand Oaks, CA: Sage.

Williams, L. J., \& McGonagle, A. K. 2016. Four research designs and a comprehensive analysis strategy for investigating common method variance with self-report measures using latent variables. Journal of Business and Psychology, 31: 339-359.

Wiltermuth, S. S. 2011. Cheating more when the spoils are split. Organizational Behavior and Human Decision Processes, 115: 157-168.

Xin, J., Chen, S., Kwan, H. K., Chiu, R. K., \& Yim, F. H. 2018. Work-family spillover and crossover effects of sexual harassment: The moderating role of work-home segmentation preference. Journal of Business Ethics, 147: 619-629.

Yang, Z., Zhang, H., Kwan, H. K., \& Chen, S. 2018. Crossover effects of servant leadership and job social support on employee spouses: The mediating role of employee organization-based self-esteem. Journal of Business Ethics, 147: 595-604.

Zhang, H., Kwan, H. K., Everett, A. M., \& Jian, Z. 2012. Servant leadership, organizational identification, and work-to-family enrichment: The moderating role of work climate for sharing family concerns. Human Resource Management, 51: 747-768.

Zhang, Y., Waldman, D. A., Han, Y.-L., \& Li, X.-B. 2015. Paradoxical leader behaviors in people management: Antecedents and consequences. Academy of Management Journal, 58: 538-566.

Zhong, C.-B., Ku, G., Lount, R. B., \& Murnighan, J. K. 2010. Compensatory Ethics. Journal of Business Ethics, 92: 323-339.

Publisher's note Springer Nature remains neutral with regard to jurisdictional claims in published maps and institutional affiliations. 
Haixiao Chen is a $\mathrm{PhD}$ candidate of human resource management at Shanghai University of Finance and Economics. His research interests include mentoring, leadership, deviant behaviors, and work-family interfaces. His work has been published in journals such as Human Resource Management, The International Journal of Human Resource Management, and Journal of Organizational Behavior.

Ho Kwong Kwan ( $\mathrm{PhD}$, Drexel University) is an associate professor of management at China Europe International Business School (CEIBS). His research interests include mentoring, leadership, deviant behavior, and work-family interfaces. His work has been published in such journals as Academy of Management Journal, Journal of Applied Psychology, Journal of Management, Journal of Management Studies, Organizational Behavior and Human Decision Processes, Personnel Psychology, Asia Pacific Journal of Human Resources, Cornell Hospitality Quarterly, Entrepreneurship Theory and Practice, Family Business Review, Human Relations, Human Resource Management, International Journal of Hospitality Management, International Journal of Human Resource Management, Journal of Business Ethics, Journal of Business Research, Journal of Management and Organization, Journal of Managerial Psychology, Journal of Occupational and Organizational Psychology, Journal of Organizational Behavior, Journal of Vocational Behavior, Leadership Quarterly, and Service Industries Journal, as well as six prior articles in Asia Pacific Journal of Management.

Jie Xin ( $\mathrm{PhD}$, Shandong University) is a professor of management at Shandong University. He is also the doctoral supervisor, postdoctoral fellow of applied economics, director of China Association of Business Ethics, and deputy director of institute of enterprise culture of Shandong University. He has published more than 40 management academic papers and 2 monographs in academic journals such as Journal of Business Ethics, Economic Management and China Soft Science. He has presided over the National Natural Science Research Foundation and The National Social Science Research Foundation, and won the SIOP Best Paper Award in the United States. 